\title{
Tool trajectory optimization of spraying robot for outer or inner-horn surface with two patches based on varied dip-angle spraying
}

\author{
Yakun Zhang ${ }^{1, a}$, Yong Zeng ${ }^{2, b}$, Dongming Liü, \\ ${ }^{1}$ Jiangsu University, Zhenjiang, China \\ ${ }^{2}$ Yancheng Institute of Technology, China \\ ${ }^{3}$ Jiangsu University, Zhenjiang, China \\ a624679583@qq.com, b475872112@qq.com, ${ }^{\mathrm{c}} 2272450178 @ q q . c o m$
}

\begin{abstract}
Keywords: outer or inner-horn surface; varied dip-angle spray; robot; trajectory optimization; coating thickness uniformity.

Abstract. To solve the problems of paint waste, poor coating thickness uniformity and low spraying efficiency for outer or inner-horn surface with two patches in the vertical spraying technology, a continuous varied dip-angle spray trajectory planning method is developed. Several problems on the off-line programming system of a spray-painting robot have been resolved, such as coating growth rate model, trajectory generation method, coating thickness models and Trajectory Optimization. The results of simulations have shown that the optimization method is feasible and effective.
\end{abstract}

\section{Introduction}

Robot spray painting is widely used in the painting operations of automobile, ship, and aerospace industries. Several in-depth studies on the off-line programming system of a spray-painting robot have been reported concerning three key problems: (i) coating growth rate modeling of a spray gun, (ii) subdivision modeling of complex free surface for spraying, and (iii) optimization algorithm of spraying trajectory.

In the aspect of coating growth rate modeling, a limited range models are widely applied to develop the coating growth rate model. For example, piecewise function model, $\beta$ distribution model, analytical deposition model, parabolic model, ellipse dual- $\beta$ model and Gaussian sum model. The mathematical expressions of these models are usually derived by means of mathematical analysis. At the same time, based on the mathematical expressions, the final model of coating growth rate is approximated by the fitting of the experimental data. These models can be widely used in the case of complicated surface shape, if these models meet the error requirement. But these models are established based on static vertical spraying experiment, the model of dip-angle spraying technology has not yet been considered.

Although the research results have been obtained in the vertical spraying process, there are still not yet overcome problems in the research. For example, in order to prevent the collision between the robot arm and the workpiece in the vertical spraying of the spray gun when spraying the surface with outer or inner-horn feature, the robot grasps the gun frequently to take the dip-angle position to carry on the spraying operation. However, scholars have not yet attached importance to the research of trajectory planning method when robot dip-angle spraying. Aiming at this kind of problem, the spray technology planning scheme is developed to select reasonable spray technology for a complex free surface with multiple patches by the horn feature recognition. A method of trajectory planning with discontinuous varied dip-angle spraying is developed to planning the spray trajectory of horn feature surface when the direction of varied dip-angle is perpendicular to the direction of trajectory planning, this method effectively improves the uniformity of coating thickness in the inner-horn place of the surface after the trajectory optimization, but in the research, the trajectory planning method of continuous varied dip-angle spray has not been given when the direction of varied dip-angle and the direction of trajectory planning are consistent. 
In this paper, a coating growth rate model of varied dip-angle spray is developed to establish the superposition model of coating thickness when the spray gun is dynamic. Optimization processes are developed to optimize the coating thickness for horn surface with two patches. By modifying the spray gun velocity and the spray height, the coating thickness deviation from the required coating thickness is optimized. Some surfaces with two patches were used to test the scheme. Simulations were performed to verify the generated trajectories. Simulations were also performed for a surface with two flat patches to verify the optimized parameters.

\section{Coating growth rate model of varied dip-angle spray}

In this paper, the coating growth rate model of varied dip-angle spray is established. Under the assumption that the spray height $H$, the spray flow $q_{v}$, and the cone angle $\varphi$ are constant during the spray gun static spraying. The distribution of the coating films formed by the vertical spraying on a flat plate approximately appears as a parabolic distribution. This can be expressed as follows:

$$
f(r)= \begin{cases}A\left(R^{2}-r^{2}\right) & |r| \leq R \\ 0 & |r|>R\end{cases}
$$

Where $R$ is the radius of spraying and $A$ is a constant; $r$ is the distance from one point $S$ on the plane to the projection point of the center of the spray gun along the spraying direction.

Assuming the small round regions are $c_{1}$ and $c_{2}$ by spraying, respectively in the reference planes P1 and $\mathrm{P} 2$ which are perpendicular to the spray direction of the spray gun, as shown in Figure $1, H_{s}$ is the distance from the nozzle to the point $S$ along the axis of the spray gun.

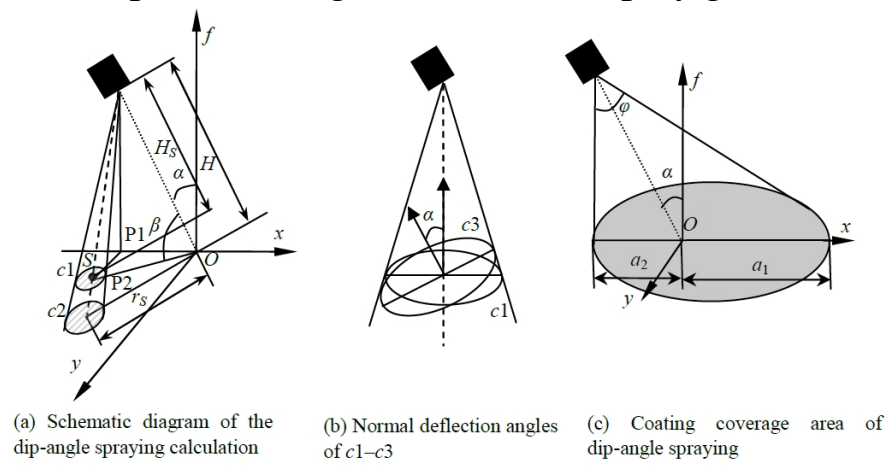

Figure 1. Schematic diagram of the coating growth rate model calculation for dip-angle spraying.

Combining formulas of differential geometry, the theoretical model of coating growth rate which takes into account the spray dip-angle as a variable can be expressed as:

$f(x, y, \alpha)=A\left(R^{2}-r_{S}^{2}\right)\left(\frac{H}{H_{S}}\right)^{2} \cos \alpha$

Where: $r_{S}=\frac{H \sin \beta \sqrt{x^{2}+y^{2}}}{H+x \sin \alpha}, \sin \beta=\sqrt{\frac{x^{2} \cos ^{2} \alpha+y^{2}}{x^{2}+y^{2}}}, H_{S}=H+x \sin \alpha$

$\alpha$ is the spray dip-angle, $r_{s}$ is the distance from the projection point of the point $S$ on P2 to the axis of the spray gun. When the long axis of the ellipse is divided into two segments $a_{1}$ and $a_{2}$ through point $O$, as shown in Figure 1, their lengths can be expressed as follows:

$$
\begin{aligned}
& \alpha_{1}(H, \alpha)=\frac{H \tan ^{2}(\varphi / 2) \sin \alpha-H \tan (\varphi / 2) \cos \alpha}{\tan (\varphi / 2) \sin (2 \alpha)-1-\tan ^{2}(\varphi / 2) \sin ^{2} \alpha+\sin ^{2} \alpha} \\
& \alpha_{2}(H, \alpha)=\frac{H \tan ^{2}(\varphi / 2) \sin \alpha+H \tan (\varphi / 2) \cos \alpha}{\tan (\varphi / 2) \sin (2 \alpha)+1+\tan ^{2}(\varphi / 2) \sin ^{2} \alpha-\sin ^{2} \alpha}
\end{aligned}
$$




\section{Trajectory design of continuous varied dip-angle spraying}

\section{Influence factors analysis of vertical spraying technology on spraying effect}

As we all know, if the number of corners is too much in the generated spray trajectory, this will have too difficult in trajectory design. Besides, if the angle between two adjacent patches gets certain value, the paint will diffuse into the air at the junction of two patches in the vertical spraying technology. In order to overcome the disadvantage of vertical spraying technology, a varied dip-angle spraying technology is used to reduce the number of corners in the trajectory and to reduce the pollution and waste of paint, respectively, as shown in Figure 2.

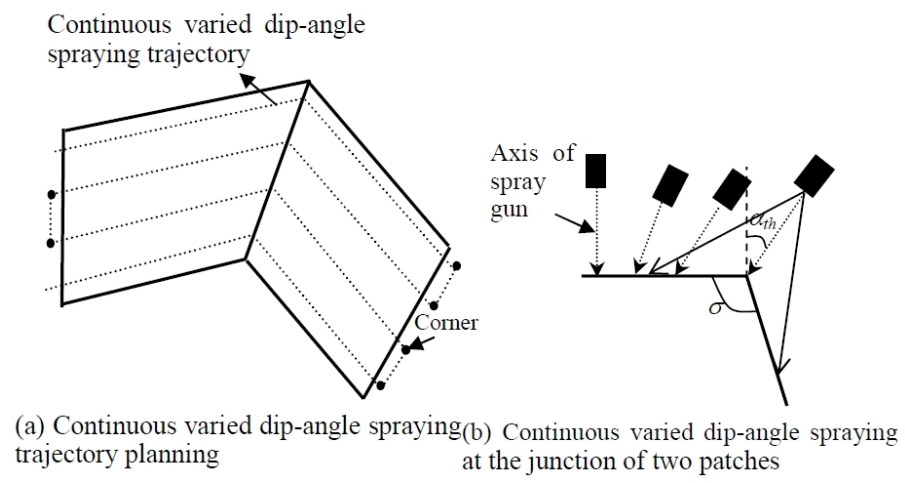

Figure 2. Continuous varied dip-angle spraying trajectory planning and spraying at the junction of two patches.

\section{Trajectory Generation of Continuous Varied Dip-angle Spraying}

The bounding-box method is usually used to just contain the surface with two patches, and a series of cutting plane which perpendicular to the intersecting line are used to cutting the surface with two patches, the distance between each cutting plane is $\delta$, there are the process of the spray gun spraying on the generated trajectory. In addition, the spray trajectory near the intersecting line is divided into three sections; they are the vertical spray trajectory, the continuous varied dip-angle spray trajectory and the constant dip-angle spray trajectory, respectively. As shown in Figure 3.

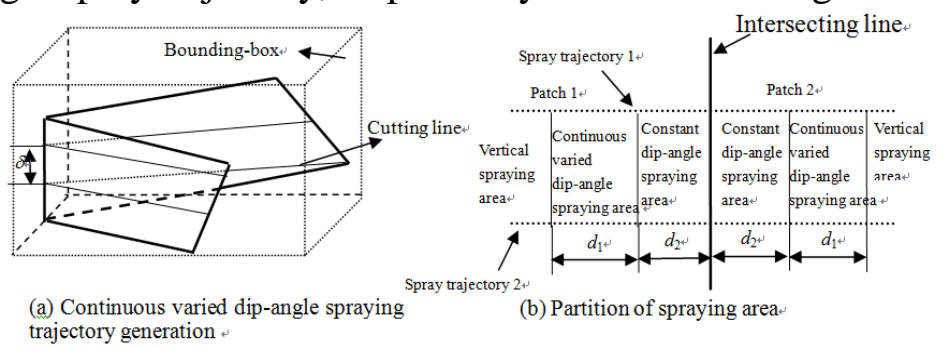

Figure 3. Continuous varied dip-angle spraying trajectory generation and partition of spraying area.

\section{Trajectory Optimization of Dip-angle Spraying}

In order to achieve the best uniformity of coating thickness in each spraying area, trajectory parameters need to be optimized. Due to the offset distance between the adjacent spray trajectory has been set. Therefore, here need to optimize the spray velocities and the corresponding spray heights on the continuous varied dip-angle spray trajectory segment and the constant dip-angle spray trajectory segment. But before optimizing the parameters, the coating thickness models in each spraying area should be established.

\section{Coating Thickness models of spray gun sprayed along each trajectory segment}

The vertical spray trajectory section, the continuous varied dip-angle spray trajectory section and the constant dip-angle spray trajectory section are respectively defined as P11 (P21), P12 (P22) and P13 (P23) on adjacent two spray trajectories. $\mathrm{P} 13^{*}$ and $\mathrm{P} 23^{*}$ are symmetric the constant dip-angle spray trajectory sections respectively with P13 and P23. The length of P12 (P22) is set to $d_{1}$; the length of $\mathrm{P} 13$ (P23) is set to $d_{2}$. As shown in Figure 4, the rectangular frame of $S_{1}$ is made up of P12 and P22, which indicate the continuous varied dip-angle spraying area, the rectangular frame of $S_{2}$ is made up of P13 and P23, which indicate the constant dip-angle spraying area. 


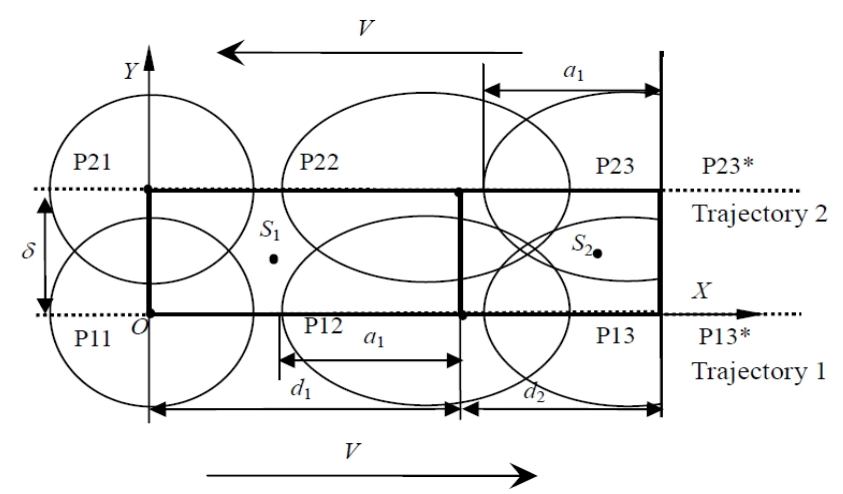

Figure 4. Schematic diagram of coating thickness calculation for varied dip-angle spraying.

P11 and P21 are the vertical spray trajectory sections, the spray velocity is set as $v_{0}$. Because the dynamic coating thickness model can be obtained by integrating the coating growth rate model, the coating thickness of the point $S_{1}$ and $S_{2}$ after spray gun spraying P11 and P21 can be expressed as:

Sections P11 and P21:

$$
T_{P 11, P 21}(x, y)=\frac{1}{v_{0}} \int_{-H_{0} \tan (\varphi / 2)}^{0} f\left(\alpha=0, \gamma_{S_{1,2}}\right) d z
$$

Where:

$$
\gamma_{S_{1,2}}=\sqrt{\left(y+y_{0}\right)^{2}+\left(z+x+H_{0} \tan (\varphi / 2)\right)^{2}}
$$

P12 and P22 are continuous varied dip-angle spray trajectory segment, in order to simplify the problem, P12 and P22 are divided into $i$ segments by using discrete method. Assuming that the spray dip-angle, the spray velocity and the spray height remain unchanged on each segment. Where the coating thickness of the point $S_{1}$ and $S_{2}$ after spray gun spraying P12 and P22 can be expressed as:

Segments P12 and P22:

$$
T_{P 12, P 22}(x, y, j)=\frac{1}{v_{j}} \int_{\frac{j-1}{i} d_{1}}^{\frac{j}{i} d_{1}} f\left(\frac{j}{i} \alpha_{t h}, \gamma_{S_{1,2}}\right) d z
$$

Where: $j \in[1, i]$, and $j$ is a positive integer

$$
\gamma_{S_{1,2}}=\frac{H_{j} \sin \beta_{j} \sqrt{\left(y+y_{0}\right)^{2}+(x-z)^{2}}}{H_{j}+(x-z) \sin \left(\frac{j}{i} \alpha_{t h}\right)}
$$

$v_{i}$ is the spray velocity, $H_{i}$ is the spraying height, $(j / i) \alpha_{t h}$ is the corresponding spray dip-angle.

P13 and P23 are constant dip-angle spray trajectory segment, the dip-angle is $\alpha_{t h}$, assuming the spray height is $H_{i+1}$ and remain unchanged. Here to optimize the spray velocity. P13 and P23 are divided into $n$ segments by using discrete method and assuming the spray velocity remains unchanged on each segment. The coating thickness of the point $S_{1}$ and $S_{2}$ after spray gun spraying P13 and P23 can be expressed as:

Segments P13 and P23:

$$
T_{P 13, P 23}(x, y)=\frac{1}{v_{i+m}} \int_{d_{1}+\frac{m-1}{n} d_{2}}^{d_{1}+\frac{m}{n} d_{2}} f\left(\alpha_{t h}, \gamma_{S_{1,2}}\right) d z
$$

Where: $m \in[1, n]$, and $m$ is a positive integer

$$
\gamma_{S_{1,2}}=\frac{H_{i+1} \sin \beta_{t h} \sqrt{\left(y+y_{0}\right)^{2}+(x-z)^{2}}}{H_{i+1}+(x-z) \sin \alpha_{t h}}
$$

$\mathrm{P} 13^{*}$ and $\mathrm{P} 23^{*}$ are constant dip-angle spray trajectory segments of the adjacent patch, $\mathrm{P} 13^{*}, \mathrm{P} 23^{*}$ and P13, P23 are respectively symmetric with respect to the intersecting line of combination patches. When the spray gun spraying on $\mathrm{P} 13^{*}$ and $\mathrm{P} 23^{*}$, part of the paint sprayed out from the spray gun that spread to the adjacent patch, so need to consider the effect of this part of the paint on the coating thickness in the constant dip-angle spraying area. Assuming spray on the patch 1 can be seen as varied spray height spray on the extension of the patch 2 . Assuming the spray height is $H_{z}$, the spray distance 
is $a_{1}\left(H_{z}, \alpha_{t h}\right)$, as shown in figure 5. $a_{1}\left(H_{z}, \alpha_{t h}\right)$ is divided into $k$ segments by using discrete method. Assuming that the spray velocity remain unchanged on each segment, the spray velocities are $v_{i+n}$, $v_{i+n-1}, \ldots, v_{i+n-k+1}$. Here regarding the usual outer horn as an example, the coating thickness of the point $S_{1}$ and $S_{2}$ after spray gun spraying $\mathrm{P} 13 *$ and $\mathrm{P} 23 *$ can be expressed as:

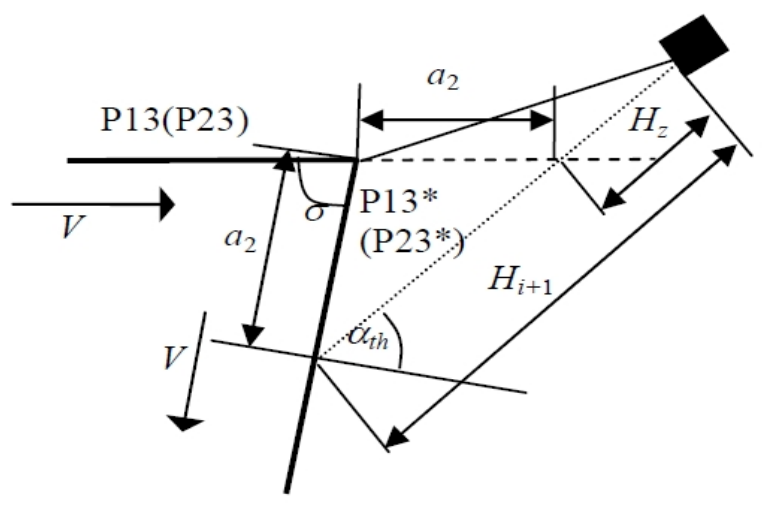

Figure 5. Schematic diagram of coating thickness calculation at the junction of two patches.

Segments $\mathrm{P} 13^{*}$ and $\mathrm{P} 23^{*}$ :

$T_{P 13^{*}, P 23^{*}}(x, y)=\frac{1}{v_{i+m}} \int_{d_{1}+d_{2}+\frac{l-1}{k} a_{1}\left(H_{z}, \alpha_{t h}\right)}^{d_{1}+d_{2}+\frac{l}{k} a_{1}\left(H_{z}, \alpha_{i h}\right)} f\left(\alpha_{t h}, \gamma_{S_{1,2}}\right) d z$

Where: $m \in[n-k+1, n], l \in[1, k], k \leq n$, and $l$ is a positive integer

$H_{z}=H_{i+1}+2 z \sin \alpha_{t h}, \gamma_{S_{2}}=\frac{H_{z} \sin \beta_{t h} \sqrt{\left(y+y_{0}\right)^{2}+(x-z)^{2}}}{H_{z}+(x-z) \sin \alpha_{t h}}$

In the formula, if the coating thickness are expressed on P11, P12, P13 and P13*, take $y_{0}=0$, if the coating thickness are expressed on $\mathrm{P} 21, \mathrm{P} 22$ and $\mathrm{P} 23^{*}$, take $y_{0}=-\delta$.

The coating thickness of the points $S_{1}$ and $S_{2}$ in the spraying areas are formed by superposition the coating thickness after spraying along the two adjacent trajectories, and the coating thickness models are influence by the values of $d_{1}$ and $d_{2}$. According to different values of $d_{1}$ and $d_{2}$, the models are expressed as follow:

$$
\begin{aligned}
& T_{S_{1}}(x, y)= \begin{cases}\sum_{j=1}^{i} T_{P 12, P 22}(x, y, j)+\sum_{m=1}^{n} T_{P 13, P 23}(x, y) & a_{2}\left(H_{\min }, \alpha_{i h}\right)<d_{2}<a_{2}\left(H_{\max }, \alpha_{t h}\right) \\
+\sum_{l=1}^{k} T_{P 3^{*}, P 3^{*}}(x, y)+T_{P 11, P 21}(x, y) & \\
\sum_{j=1}^{i} T_{P 12, P 22}(x, y, j) & d_{2} \geq a_{2}\left(H_{\max }, \alpha_{t h}\right) \\
+\sum_{m=1}^{n} T_{P 13, P 23}(x, y)+T_{P 11, P 21}(x, y) & \end{cases} \\
& T_{S_{2}}(x, y)= \begin{cases}\sum_{j=1}^{i} T_{P 12, P 22}(x, y, j)+\sum_{m=1}^{n} T_{P 13, P 23}(x, y) & 0<d_{1}<H_{0} \tan (\varphi / 2) \\
+\sum_{l=1}^{k} T_{P 13^{*}, P 23^{*}}(x, y)+T_{P 11, P 21}(x, y) & d_{1} \geq H_{0} \tan (\varphi / 2) \\
\sum_{j=1}^{i} T_{P 12, P 22}(x, y, j) & \\
+\sum_{m=1}^{n} T_{P 13, P 23}(x, y)+\sum_{l=1}^{k} T_{P 13^{*}, P 23^{*}}(x, y)\end{cases}
\end{aligned}
$$

\section{Optimization of Trajectory Parameters}

In order to achieve the best coating thickness uniformity in two spraying areas, the optimization objective function is established by minimizing the variance between the coating thickness at any point within the spray area and the ideal coating thickness, to optimize the values of the spray velocity and the spray height. As follows: 


$$
\left\{\begin{array}{l}
\min E=E_{1}+E_{2} \\
E_{1}\left(v_{1}, \mathrm{~L}, v_{i}, v_{i+1}, \mathrm{~L}, v_{i+n}, H_{1}, H_{2}, \mathrm{~L}, H_{i}, H_{i+1}\right)=\int_{0}^{d_{1}} \int_{0}^{\delta}\left[T_{S_{1}}(x, y)-T_{d}\right]^{2} d x d y \\
E_{2}\left(v_{1}, \mathrm{~L}, v_{i}, v_{i+1}, \mathrm{~L}, v_{i+n}, H_{1}, H_{2}, \mathrm{~L}, H_{i}, H_{i+1}\right)=\int_{d_{1}}^{d_{1}+d_{2}} \int_{0}^{\delta}\left[T_{S_{2}}(x, y)-T_{d}\right]^{2} d x d y \\
\text { s.t. } H \in\left[H_{\text {min }}, H_{\max }\right], v \leq V_{\max }
\end{array}\right.
$$

Where $H_{\min }$ and $H_{\max }$ are the maximum and minimum values of the spray height respectively; $V_{\max }$ is the maximum spray velocity; $T_{d}$ is the ideal coating thickness.

\section{Simulation and Analysis}

According to acknowledge of experiment, we can set the related parameters. The coating thickness models in the areas of the continuous varied dip-angle spray and the constant dip-angle spray are established by the formula. The coating thickness model of any point in the continuous varied dip-angle spray area and the constant dip-angle spray area are represented respectively by the lower expressions of the formula. Finally, Parameters to be solved, according to the formula, the pattern search method is used to solve the problem in MATLAB2010 toolbox.

In order to clearly show the coating distribution in the spraying area between adjacent trajectories after optimization of spray trajectory parameters, here in the y direction to select a number of specific locations and the coating thickness of sampling points are calculated along the $\mathrm{x}$ direction of these specific locations. The coating thickness of the discrete points are collected along the $\mathrm{x}$ direction at the $\mathrm{y}=0,10.8,30.4 \mathrm{~mm}$ locations, respectively, as shown in Figure 6.

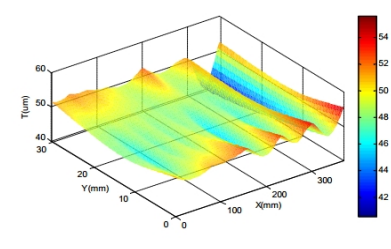

Figure 6(a). Coating thickness distribution in the spray area after trajectory optimization.

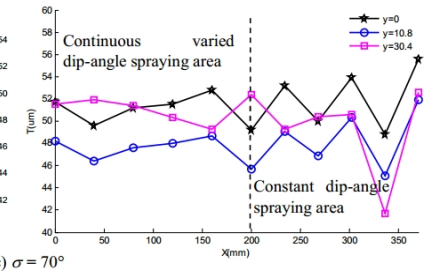

Outer

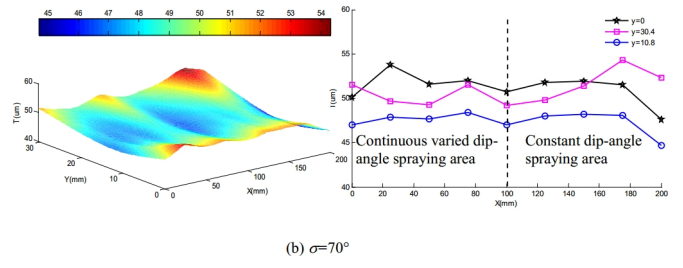

Figure 6(b). Coating thickness distribution in the spraying painting area after optimization. Inner

It can be known form the Figure 6, the maximum value and minimum value of coating thickness often appear in near the vertex of outer or inner-horn. The maximum and minimum coating thickness after spray trajectory optimization compared with the ideal value, the error values are no more than $\pm 10 \mu \mathrm{m}$ and meet the requirement of the conventional thickness uniformity. But with the decrease the angle of outer or inner-horn, increasing the coating thickness error, so the surface of the workpiece shape should be avoided in the use of smaller angle of outer or inner-horn.

\section{Conclusions}

The simulation results show that the trajectory planning method and the optimization method are feasible and effective for the continuous varied dip-angle spraying; the coating thickness uniformity can reach the best after spray parameters optimization, which can satisfy the original design goal. At the same time, it is reasonable to use the continuous varied dip-angle spray trajectory planning to reduce the number of corners in the vertical spray trajectory, which can improve the spraying efficiency.

\section{Acknowledgements}

The project was supported by the National Natural Science Foundation of China (Grant No. 51405418), the Natural Science Foundation of the Jiangsu Higher Education Institutions of China (Grant No. 14KJB460031). 


\section{References}

[1] S. R. Yu, L. G. Cao, Modeling and prediction of paint film deposition rate for robotic spray painting, Proc. 2011 IEEE International Conference on Mechatronics and Automation, Beijing, China, 2011,1445-1450.

[2] B. Zhou, Z. H. Shao, Z. D. Meng, X. Z. Dai, Gaussian sum based coat growth rate modeling of spray painting robots, Journal of Huazhong University of Science and Technology(Natural Science Edition), 41(S1), 2013, 463-466.

[3] Y. Zeng, Y. K. Zhang, J. X. He, H. Zhou, C. W. Zhang, L. Zheng, Prediction model of coating growth rate for varied dip-angle spraying based on gaussian sum model, Mathematical Problems in Engineering, 2016(2016), article no. 9369047.

[4] H. P. Chen and N. Xi, Automated robot tool trajectory connection for spray forming process,Journal of Manufacturing Science and Engineering, 134(2), 2012, article no. 021017.

[5] P. Zhang, J. Gong, H. F. Ning, Y. Zeng, Y. J. Liu, L. N. Wei, Study on trajectory combination and connection problems of spray-painting robot for large curvature combination surfaces, Journal of Sichuan University (Engineering Science Edition), 48(4), 2016, 217-222.

[6] C. M. Li, J. Gong, W. C. Niu, C. Wang, Combinatorial optimization of spray painting robot tool trajectory based on improved membership cloud models ant colony algorithm, Journal of Shanghai Jiaotong University, 49(3), 2015, 387-391.

[7] Y. Zeng, H. Zhou, C. W. Zhang, R. H. Ma and L. Zheng, Spray technology planning of spray painting robot for complex free surface, International Journal of Robotics and Automation, 31(4), 2016, 321-328.

[8] Y. Zeng, J. Gong, N. Xu et al, Spray-painting trajectory optimization of painting robot for corner 20 surfaces, Journal of Zhejiang University (Engineering Edition), 48(5), 2014, 791-798.

[9] W. Chen, D.A. Zhao, and F.Z. Li, Tool trajectory planning of robotic spray painting and its experiment for complex curved surfaces, Transactions of the Chinese Society for Agricultural Machinery, 42(1), 2011, 204-208.

[10]W. Chen and D.A. Zhao. Path planning for spray painting robot of workpiece surfaces, Mathematical Problems in Engineering, 2013(2013), 2013, article no. 659457.

[11]F. Z. Li, D. A. Zhao, and C. Zhang, Trajectory optimization of spray painting robot based on CAD, Transactions of the Chinese Society for Agricultural Machinery, 41(5), 2010, 213-217.

[12]H.P. Chen and N. Xi, Automated tool trajectory planning of industrial robots for painting composite surfaces, International Journal of Advanced Manufacturing Technology, 35(1), 2008, 680-696.

[13]H.P. Chen, T. Fuhlbrigge, and X.Z. Li, A review of CAD-based robot path planning for spray painting, Industrial Robot, 36(1), 2009, 45-50. 